\title{
基于多面体与平行入射光的光辐射源空间定向 方法分析
}

王江*

成都信息工程学院, 中国气象局大气探测重点开放实验室, 成都 610225

*E-mail: rivers2000@126.com

收稿日期: 2012-03-20; 接受日期: 2012-08-03

摘要光辐射源空间定向在航天、军事等领域一直是研究热点. 现有研究主要集中 在局部视场上的高精度定向，对 $180^{\circ}$ 以上视场的定向目前需用多个局部视场组合实 现, 导致探测系统体积、重量、功耗和成本增加问题. 定义光辐射源的辐射能量和方向 为矢量, 根据辐射余弦定理和矢量定律, 推导出了该矢量能用法矢量不共面的 3 个面 的单位法矢量及光辐射源投射在这些面上的能量来求解的结论, 以及该矢量的数学求 解公式; 基于此结论, 利用光的直线传播特性和多面体的几何特性, 提出了用多面体 实现光辐射源空间 $360^{\circ}$ 全视场定向的方法; 给出了抗多径干扰的方法; 推导出了误差 上限的计算公式, 解决了误差范围的确定问题. 实测与仿真结果验证了该方法的正确 性和可行性, 以及误差分析结果的正确性. 当 3 个测量面的单位法矢量构成的矩阵正 交, 任一测量面上能量的测量误差值与其真实值的比值上限为 $5 \%$ 或 $1 \%$ 时, 其精度优 于 $2.866^{\circ}$ 或 $0.574^{\circ}$.

关键词

光辐射源定向

辐射能量

单位法矢量

多面体

\section{1 引言}

光辐射源空间定向在许多技术领域中有着广泛 应用，如在航天应用中通过测量太阳角以实现对卫 星姿态的控制或对航天登陆设备的辅助定位 ${ }^{[1]}$; 在军 事应用中通过对激光、红外等光辐射源的被动定向以 实现预警 $[2,3]$ 等.

近年来, 随着应用发展对测量视场和精度要求 的日益提高, 国内外研究了不少大视场、高精度的光 辐射源定向方法, 尤其是在航天应用中, 如基于 CMOS APS 面阵等图像传感器对太阳定向的方法 ${ }^{[4 \sim 9]}$, 以及利用光学非线性补偿 ${ }^{[10]}$ 、游标卡尺 ${ }^{[11]}$ 和梯形狭
缝式 ${ }^{[12]}$ 等其它方式对太阳定向的方法. 这些研究主 要聚焦于 $180^{\circ}$ 视场内如何提高测量视场和精度, 受 实现原理限制, 它们的测量视场小于 $180^{\circ}$, 如文献[5] 采用 CMOS APS 面阵图像传感器对太阳定向, 视场 达到了 $\pm 64^{\circ}$ 左右, 精度优于 $0.04^{\circ}$, 文献 [6,7]通过对 数字式太阳敏感器光学系统的改进来增大测量视场 和提高精度, 且文献 [6]达到了 $120^{\circ} \times 180^{\circ}$ 的视场和 $0.02^{\circ}$ 的精度, 但因 APS 像元数目有限及面阵探测器 与孔的高度大于 $0^{[8,9]}$, 其视场均小于 $180^{\circ}$. 然而, 实 际应用中, 还存在探测视场要求超 $180^{\circ}$, 而探测精度 要求相对不高的应用. 比如卫星初始入轨或因故障 丢失原有姿态时对日的重定向, 此时, 如何实现对日 
快速、准确定向以保证卫星能源的稳定供应显得尤其 重要, 与卫星姿态控制的要求相比, 该应用对精度要 求相对不高, 但探测视场必须达 $360^{\circ}$, 可是, 现有方 法测量视场小于 $180^{\circ}$, 要实现全天球 $360^{\circ}$ 视场上对太 阳的定向, 至少需用 3 个以上探测器(太阳敏感器) 来组合实现, 这使得卫星系统体积、重量、功耗和 成本增加, 不符合当前卫星小型化、低功耗的发展 要求 ${ }^{[13,14]}$. 因此, 研究 $360^{\circ}$ 全视场测量、精度较高 且易实现的定向方法以解决此类问题十分必要.

本文基于多面体各面的单位法矢量及光辐射源 投射在这些面上的能量, 提出了一种 $360^{\circ}$ 全视场测 量、精度较高且易实现的定向方法, 推导出了该方法 的通用数学求解式及误差关系式, 解决了误差范围 难确定的问题, 并针对多径干扰给出了抗干扰方法.

\section{2 光辐射源空间定向原理及方法}

假定光辐射源的辐射光线满足相互平行或相互 间的角度很小、近似平行的条件. 定义光辐射源矢量 的方向与光辐射源辐射方向相反, 模为光辐射源投 射在与辐射方向垂直平面上的单位面积的辐射能量.

如图 1 所示, 设 $S$ 为空间任一光辐射源矢量, $n$ 为空间任一平面 $P$ 的法矢量, $S$ 与 $n$ 的夹角 $\angle(S, n)$ 为 $\Phi, S_{\perp}$ 为 $\boldsymbol{S}$ 在 $\boldsymbol{n}$ 上的射影矢量, 由射影矢量定义, 得:

$\boldsymbol{S}_{\perp}=|\boldsymbol{S}| \cos \angle(\boldsymbol{S}, \boldsymbol{n}) \boldsymbol{n}^{\circ}=(|\boldsymbol{S}| \cos \Phi) \boldsymbol{n}^{\circ}=\left|\boldsymbol{S}_{\perp}\right| \boldsymbol{n}^{\circ}$,

式中 $n^{\circ}$ 为平面 $P$ 的单位法矢量. 另外, 根据辐射余 弦定理 ${ }^{[15]}$ ——任意一个表面上的辐照度随该表面法 线和辐射能传输方向之间夹角的余弦而变化, 可得 光辐射源投射在平面 $P$ 上的单位面积的辐射能量为 $|S| \cos \Phi$, 对比(1)式可知, 光辐射源投射在平面 $P$ 上 的单位面积的辐射能量与射影矢量 $S_{\perp}$ 的模相等,

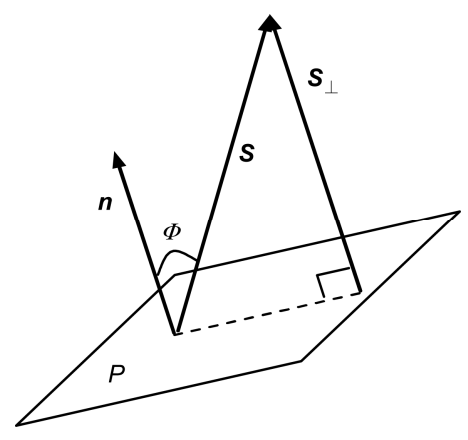

图 1 光辐射源矢量与平面关系
由此得推论一.

光辐射源矢量在平面法矢量上的射影矢量可用 光辐射源投射在该平面上的单位面积的辐射能量及 该平面的单位法矢量来表示, 其模为光辐射源投射 在该平面上的单位面积的辐射能量, 方向为平面单 位法矢量方向.

设矢量 $\boldsymbol{r}_{1}, \boldsymbol{r}_{2}, \boldsymbol{r}_{3}$ 不共面, 空间直角坐标系上任一 矢量 $\boldsymbol{r}$ 在 $\boldsymbol{r}_{1}, \boldsymbol{r}_{2}, \boldsymbol{r}_{3}$ 矢量上的射影矢量分别为 $\boldsymbol{e}_{1}, \boldsymbol{e}_{2}, \boldsymbol{e}_{3}$, 令 $e_{i}=\left|e_{i}\right| e_{i}^{\circ}(i=1,2,3)$, 其中 $\left|e_{i}\right|$ 为 $e_{i}$ 的模, $e_{i}^{\circ}$ 为 $e_{i}$ 的 单位坐标矢量, $e_{i}^{\circ}=\left(\cos \alpha_{i}, \cos \beta_{i}, \cos \gamma_{i}\right), \quad \alpha_{i}, \beta_{i}$, $\gamma_{i}$ 分别为 $e_{i}$ 的方向角, 由 $\boldsymbol{r}$ 与 $\boldsymbol{e}_{1}, \boldsymbol{e}_{2}, \boldsymbol{e}_{3}$ 的关系有:

$$
e_{i}^{\circ} \cdot r=\left|e_{i}\right| \text {. }
$$

设 $\boldsymbol{r}=(x, y, z)^{\mathrm{T}}$, 由(2)式得方程:

$$
x \cos \alpha_{i}+y \cos \beta_{i}+z \cos \gamma_{i}=\left|e_{i}\right|,
$$

其中 $i$ 为 $1,2,3$. 令 $\boldsymbol{A}=\left[\begin{array}{ccc}\cos \alpha_{1} & \cos \beta_{1} & \cos \gamma_{1} \\ \cos \alpha_{2} & \cos \beta_{2} & \cos \gamma_{2} \\ \cos \alpha_{3} & \cos \beta_{3} & \cos \gamma_{3}\end{array}\right], \boldsymbol{e}=$ $\left[\begin{array}{l}\left|e_{1}\right| \\ \left|e_{2}\right| \\ \left|e_{3}\right|\end{array}\right]$, 得:

$$
\boldsymbol{A r}=\boldsymbol{e} .
$$

因 $\boldsymbol{r}_{1}, \boldsymbol{r}_{2}, \boldsymbol{r}_{3}$ 不共面, 得 $\boldsymbol{e}_{1}, \boldsymbol{e}_{2}, \boldsymbol{e}_{3}$ 互不相关, 因此 $|A| \neq 0, \boldsymbol{A}$ 存在逆矩阵 $\boldsymbol{A}^{-}$. 将(4)式两端左乘 $\boldsymbol{A}^{-}$, 得:

$$
\boldsymbol{r}=\boldsymbol{A}^{-} \boldsymbol{e} .
$$

矢量 $\boldsymbol{r}$ 在空间直角坐标系上的方向余弦为:

$\cos \alpha=x /|r|, \quad \cos \beta=y /|r|, \quad \cos \gamma=z /|r|$,

其中, $\alpha, \beta, \gamma$ 为矢量 $\boldsymbol{r}$ 的方向角, $|r|=\sqrt{x^{2}+y^{2}+z^{2}}$.

在空间直角坐标系上, 假设 $\boldsymbol{r}$ 为光辐射源矢量, $\boldsymbol{r}_{1}, \boldsymbol{r}_{2}, \boldsymbol{r}_{3}$ 为 3 个平面的法矢量, 由上述推导可知, 当 3 个平面的法矢量不共面时, 光辐射源矢量可由它在 这 3 个平面的法矢量上的射影矢量通过公式(5)求得, 而光辐射源空间方向可用光辐射源矢量通过公式(6) 求得. 结合推论一, 得: 在空间直角坐标系上, 光辐 射源矢量可由光辐射源投射在 3 个法矢量不共面的 平面上的单位面积的辐射能量及这 3 个面的单位法 矢量通过公式(5)求得, 而光辐射源空间方向可由光 辐射源矢量通过公式(6)求得.

进一步地, 将光辐射源照射对象扩展为由多个 平面组成的多面体, 此时, 对于空间任意方向的光辐 
射源, 当多面体的几何结构满足: 存在至少 3 个以上 被光辐射源同时直射且法矢量不共面的面, 则光辐 射源矢量可由光辐射源投射在多面体各面上的单位 面积的辐射能量及多面体各面的单位法矢量通过公 式(5)求得, 而光辐射源空间方向可由光辐射源矢量 通过公式(6)求得.

通常, 为实现 $360^{\circ}$ 全景视场的测量, 多面体的面 数须大于 5. 为避免因安装造成的测量视场遮挡问题, 可用被安装物体的外表为测量多面体来实现 $360^{\circ}$ 全 视场测量, 如在航天器表面选择光传感器的安装面, 以这些安装面构成的多面体来实现 $360^{\circ}$ 全视场探测.

实际应用中, 当光辐射源与测量多面体距离足 够远时, 光辐射源照射在多面体上的光线可近似看 作平行, 此时, 如果通过光传感器从多面体面上测量 得到的单位面积的辐射能量满足辐射余弦定理, 光 传感器对辐射能量的转化率为常数 $\eta(\eta \leqslant 1)$, 则光辐 射源矢量为 $\eta \boldsymbol{r} . \eta \boldsymbol{r}$ 与 $\boldsymbol{r}$ 同向, 可见, 转化率 $\eta$ 与光辐 射源空间方向无关, 由此, 光辐射源矢量的计算式可 简化为:

$$
\boldsymbol{r}=\boldsymbol{A}^{-} \boldsymbol{S},
$$

式中矢量 $\boldsymbol{S}=\left[\begin{array}{lll}s_{1} & s_{2} & s_{3}\end{array}\right]^{\mathrm{T}}$, 它由多面体上 3 个不共 面的面上测量得到的单位面积的辐射能量 $s_{1}, s_{2}$ 和 $s_{3}$ 构成.

\section{3 抗多径干扰方法与分析}

因测量环境不理想, 实际测量环境中常存在光 辐射源多径传输产生的干扰, 如在卫星上测太阳入 射角时存在地球反照等太阳多径干扰, 此时, 从多面 体面上测得的辐射能量将包含这些干扰分量, 导致 光辐射源定向出现偏差.

根据光辐射源定向原理, 当多面体上存在 3 个以 上被光辐射源同时直射且法矢量不共面的平面时, 只要这些面中存在 3 个未被干扰且法矢量不共面的 面, 则能测得光辐射源矢量的真实值. 进一步地, 根 据光辐射源的直线传播特性, 由解析几何可推得: 当 多径干扰源辐射方向照射的球面不能完全覆盖待测 光辐射源辐射方向照射的球面时, 则在与球体无限 接近的多面体上必定存在 3 个以上仅被待测光辐射 源同时直射且法矢量不共面的面. 因此, 增加多面体 上被任意方向光辐射源同时直射的几何面, 使其被
光辐射源同时直射的面中至少存在 3 个以上未被多 径干扰源照射且法矢量不共面的面, 找出这些未被 干扰的面, 则可测得光辐射源矢量的真实值, 从而消 除多径干扰源对测量的影响.

\section{4 误差分析}

受光辐射源多径传输及制造工艺水平限制, 多 面体面上光传感器实测得到的单位面积的辐射能量 为:

$$
s=s_{\mathrm{d}}+\underbrace{\varepsilon_{\text {位置 }}+\varepsilon_{\text {传感器 }}+\varepsilon_{\text {多径 }},}_{\varepsilon}
$$

式中, $s_{\mathrm{d}}$ 为光辐射源直射光投射到该面的单位面积的

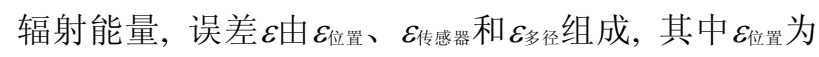

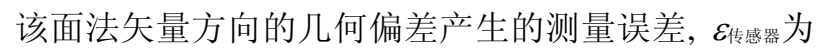
该面光传感器的输出偏差产生的测量误差, $\varepsilon$ 多径为由 光辐射源多径传输产生的光辐射源非直射光投射在

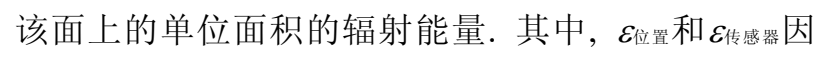
测量装置不理想产生, 为测量装置固有误差, 它决定 了测量装置理论上的最高测量精度; 而 $\varepsilon$ 多径是由外部 环境产生的误差, 其来源、大小和分布随环境变化.

假设测量无误差, 由(4)式可得光辐射源矢量 $\boldsymbol{r}$ 与光辐射源投射到多面体 3 个不共面的面上单位面 积的辐射能量构成的矢量 $\boldsymbol{S}$ 间的关系为:

$$
\boldsymbol{A r}=\boldsymbol{S} .
$$

当 $S$ 存在扰动误差 $\Delta S$ 时, 上式变为:

$$
\boldsymbol{A}(\boldsymbol{r}+\Delta \boldsymbol{r})=\boldsymbol{S}+\Delta \boldsymbol{S},
$$

其中, $\Delta \boldsymbol{r}$ 为由 $\Delta \boldsymbol{S}$ 扰动产生的光辐射源矢量的误差矢 量, 为:

$$
\Delta r=A^{-} \Delta S
$$

由(11)式, 有:

$$
\|\Delta r\|=\left\|A^{-} \Delta S\right\| \leqslant\left\|A^{-}\right\|\|\Delta S\| .
$$

由(9)式得 $\frac{1}{\|\boldsymbol{r}\|} \leqslant \frac{\|\boldsymbol{A}\|}{\|S\|}$, 结合(12)式, 得:

$$
\frac{\|\Delta \boldsymbol{r}\|}{\|\boldsymbol{r}\|} \leqslant\left\|A^{-}\right\|\|\boldsymbol{\| A}\| \frac{\|\Delta S\|}{\|S\|}=\operatorname{Cond}(A) \frac{\|\Delta S\|}{\|S\|},
$$

其中 $\operatorname{Cond}(\boldsymbol{A})$ 为矩阵 $\boldsymbol{A}$ 的条件数, $\operatorname{Cond}(\boldsymbol{A}) \geqslant 1$, 当 $\operatorname{Cond}(A) \neq 1$ 时, 对同一 $\Delta S, \Delta r$ 将被矩阵 $\boldsymbol{A}$ 放大. 下面 结合光辐射源矢量与其误差矢量在空间直角坐标系 上的几何关系，分析由 $\Delta S$ 扰动产生的光辐射源空间 方向误差. 
令实测光辐射源矢量为 $\boldsymbol{r}^{\prime}, \boldsymbol{r}^{\prime}=\boldsymbol{r}+\Delta \boldsymbol{r}$, 定义矢量 $\boldsymbol{r}^{\prime}$ 与 $\boldsymbol{r}$ 的夹角 $\theta$ 来表示光辐射源空间方向误差. $\boldsymbol{r}^{\prime}, \boldsymbol{r}, \Delta \boldsymbol{r}$ 和 $\theta$ 之间的几何关系如图 2 所示, 夹角 $\theta$ 的大小随矢 量 $\Delta \boldsymbol{r}$ 变化, 当 $\Delta \boldsymbol{r}$ 与 $\boldsymbol{r}^{\prime}$ 垂直时, 对相同的 $|\Delta \boldsymbol{r}|, \theta$ 最大, 记为 $\theta_{\max }$; 当 $\Delta \boldsymbol{r}$ 与 $\boldsymbol{r}$ 正向或反向的夹角很小时, 即使 $|\Delta \boldsymbol{r}|$ 较大, $\theta$ 也很小, 特别是当 $\Delta \boldsymbol{r}$ 与 $\boldsymbol{r}$ 正向或反向的方 向相等时, $\theta$ 为 0 ; 当 $\Delta \boldsymbol{r}$ 与 $\boldsymbol{r}$ 的夹角 $\angle(\Delta r, r)<90^{\circ}$ 时, $\theta<\theta_{\text {max }} . \boldsymbol{r}, \Delta \boldsymbol{r}, \theta$ 和 $\theta_{\text {max }}$ 之间的关系为:

$$
\theta \leqslant \theta_{\text {max }}=\arcsin (|\Delta \boldsymbol{r}| /|\boldsymbol{r}|) \text {. }
$$

因 $|\Delta \boldsymbol{r}| /|\boldsymbol{r}|=\|\Delta \boldsymbol{r}\|_{2} /\|\boldsymbol{r}\|_{2}$, 由(13), (14)式, 得 $\theta$ 与矩 阵 $\boldsymbol{A}, \Delta \boldsymbol{S}$ 和 $\boldsymbol{S}$ 的关系为:

$\theta \leqslant \arcsin \left(\operatorname{Cond}(\boldsymbol{A})_{2} \frac{\|\Delta S\|_{2}}{\|S\|_{2}}\right)=\arcsin \left(\operatorname{Cond}(\boldsymbol{A})_{2} \frac{|\Delta S|}{|\boldsymbol{S}|}\right)$.

由(15)式可知, 光辐射源矢量实测与真实方向的 偏差上限依赖于矩阵 $\boldsymbol{A}$ 和矢量 $\boldsymbol{S}$ 与其扰动误差矢量 $\Delta S$ 的模. 当矩阵 $\boldsymbol{A}$ 正交时, 即 3 个面的法矢量互相垂 直, 此时, $\operatorname{Cond}(\boldsymbol{A})=1, \theta$ 的上限与矩阵 $\boldsymbol{A}$ 无关, (15)式 简化为:

$$
\theta \leqslant \arcsin (|\Delta S| /|S|) .
$$

因矩阵 $\boldsymbol{A}$ 正交, 公式(9)为空间直角坐标系的转 轴公式, 矢量 $\boldsymbol{S}$ 和 $\Delta \boldsymbol{S}$ 为矢量 $\boldsymbol{r}$ 和 $\Delta \boldsymbol{r}$ 通过坐标旋转变 换而得, 根据坐标旋转变换特性, $S, \Delta S$ 和 $S+\Delta S$ 间的 几何关系与矢量 $\boldsymbol{r}, \Delta \boldsymbol{r}$ 和 $\boldsymbol{r}+\Delta \boldsymbol{r}$ 保持不变, 因此, 矢量 $\boldsymbol{r}+\Delta \boldsymbol{r}$ 与 $\boldsymbol{r}$ 的夹角与矢量 $\boldsymbol{S}+\Delta \boldsymbol{S}$ 与 $\boldsymbol{S}$ 的夹角相等.

\section{5 实验与分析}

\section{1 仿真与结果分析}

设 $\Delta \boldsymbol{S}=\left[\begin{array}{lll}k_{1} s_{1} & k_{2} s_{2} & k_{3} s_{3}\end{array}\right]^{\mathrm{T}}$, 其中 $\boldsymbol{S}=\left[\begin{array}{lll}s_{1} & s_{2} & s_{3}\end{array}\right]^{\mathrm{T}}$,

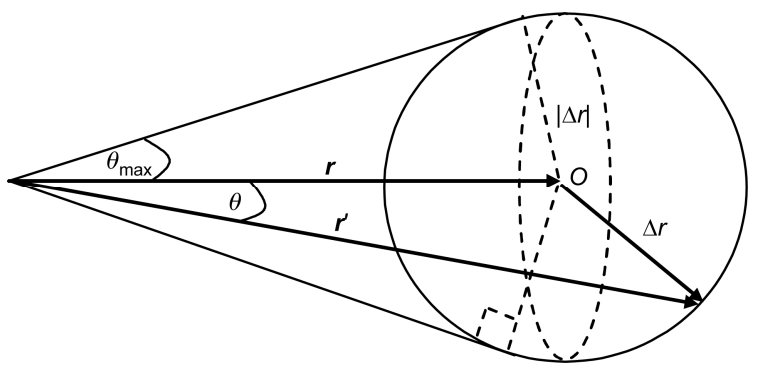

图 2 光辐射源矢量实测与真实方向几何关系 $\boldsymbol{k}=\left(k_{1}, k_{2}, k_{3}\right)$. 假设光辐射源的辐射能量恒定，在空间 直角坐标系 $x o y$ 平面和 $z$ 正轴构成的空间内, 光辐射 源以 $30^{\circ}, 60^{\circ}$ 和 $80^{\circ}$ 的高度角分别按方位角从 $0^{\circ} \sim 360^{\circ}$ 的轨迹变化. 由 3 个平面的单位法矢量构成的矩阵 $\boldsymbol{A}$ 分别为: 非正交矩阵

$$
\left[\begin{array}{ccc}
0 & 0 & 1 \\
0.9636 & 0 & 0.2672 \\
0 & 0.9636 & 0.2672
\end{array}\right]
$$

和正交矩阵组

$$
\left[\begin{array}{lll}
0 & 0 & 1 \\
1 & 0 & 0 \\
0 & 1 & 0
\end{array}\right],\left[\begin{array}{ccc}
0 & 0 & 1 \\
-1 & 0 & 0 \\
0 & 1 & 0
\end{array}\right],\left[\begin{array}{ccc}
0 & 0 & 1 \\
-1 & 0 & 0 \\
0 & -1 & 0
\end{array}\right],\left[\begin{array}{ccc}
0 & 0 & 1 \\
1 & 0 & 0 \\
0 & -1 & 0
\end{array}\right],
$$

正交矩阵组中各矩阵的方位角探测范围为 $90^{\circ}$, 它们 通过组合实现 $360^{\circ}$ 方位角探测. $\Delta S$ 通过选择不同 $\boldsymbol{k}$ 值, 由式 $\Delta \boldsymbol{S}=\left[\begin{array}{lll}k_{1} s_{1} & k_{2} s_{2} & k_{3} s_{3}\end{array}\right]^{\mathrm{T}}$ 得到.

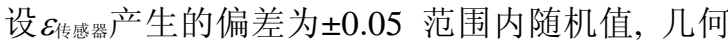
位置偏差为 $\pm 1^{\circ}$, 假定光辐射源矢量与平面法线夹角

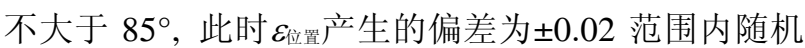
值, $\varepsilon$ 多径产生的偏差为 $[0,0.1]$ 范围内随机值. 随着光

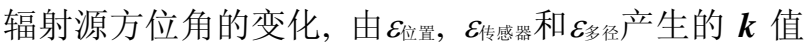
如图 3 所示, 图 3(a), (b)和(c)分别为 $k_{1}, k_{2}, k_{3}$ 在不同 方位角上的值. 采用图 3 的 $\boldsymbol{k}$ 值，图 4 和 5 给出了对 非正交矩阵和正交矩阵的仿真结果，其中，图 4(a)和 图 5(a)为实际误差曲线, 图 4(b)和图 5(b)为误差上限 曲线, 图 4(c)和图 5(c)为误差上限与实际误差的差值 曲线. 由图 4 和 5 可见, 两者的实际误差都不大于它 们的误差上限, 非正交矩阵的误差上限明显大于正 交矩阵, 而且, 非正交矩阵实际误差的最大值也明显 大于正交矩阵，验证了误差关系式的正确性. 同样, 采用图 3 的 $\boldsymbol{k}$ 值, 图 6 给出了非正交矩阵和正交矩阵 得到的矢量 $S+\Delta S$ 和 $S$ 夹角与矢量 $\boldsymbol{r}+\Delta \boldsymbol{r}$ 和 $\boldsymbol{r}$ 夹角之 差的仿真结果, 其中图 6(a)为非正交矩阵的仿真结果, 图 6(b)为正交矩阵的仿真结果. 由图 6 可见, 对于正 交矩阵, 矢量 $\boldsymbol{r}+\Delta \boldsymbol{r}$ 与 $\boldsymbol{r}$ 的夹角等于矢量 $\boldsymbol{S}+\Delta \boldsymbol{S}$ 与 $\boldsymbol{S}$ 的 夹角, 矩阵变换不影响矢量 $\boldsymbol{r}+\Delta \boldsymbol{r}$ 与 $\boldsymbol{r}$ 的几何关系, 而 对于非正交矩阵则不成立.

图 7 为以 $\boldsymbol{k}=(0.08,0.1,0.1)$ 为中心, $\boldsymbol{k}$ 的各分量在 \pm 0.01 内随机扰动时采用非正交矩阵得到的实际误差 和误差上限的仿真结果. 对比图 4 和 7 可知, 两者误 差上限都很大，但对同一高度角，图 7 的实际误差 

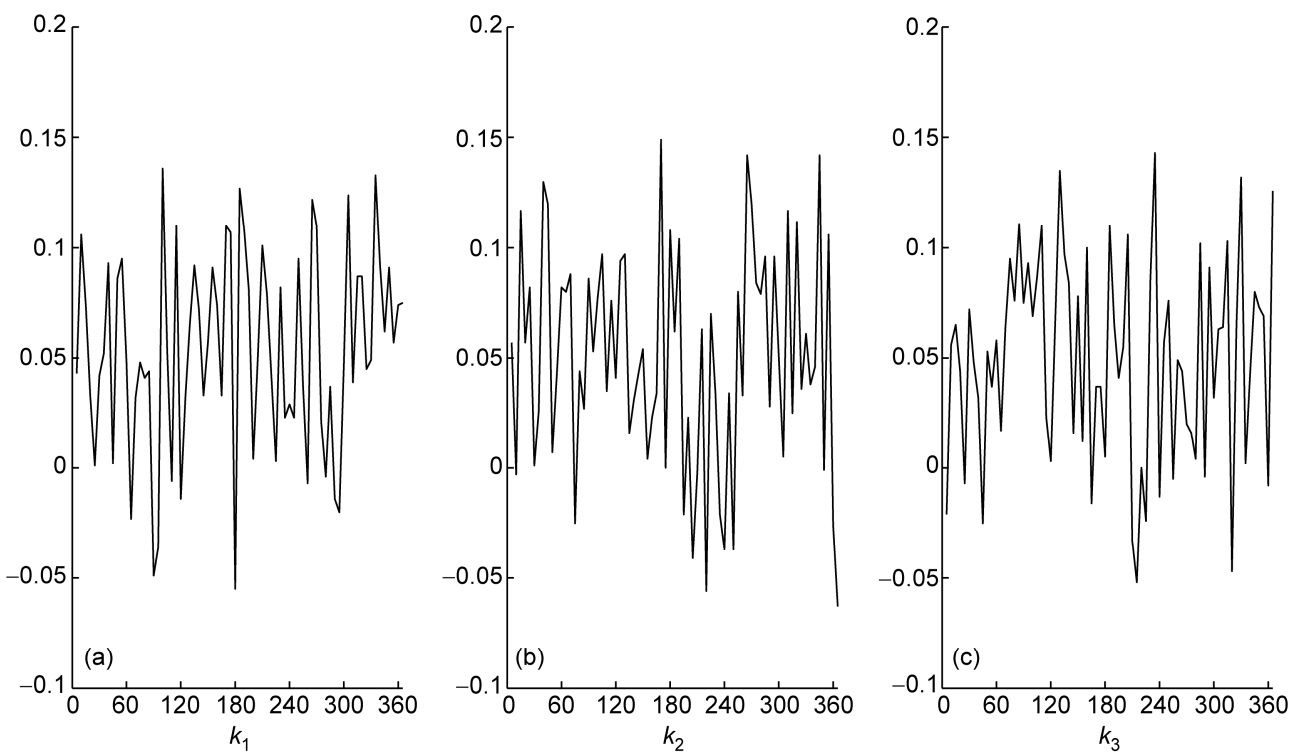

图 $3 k$ 值仿真曲线
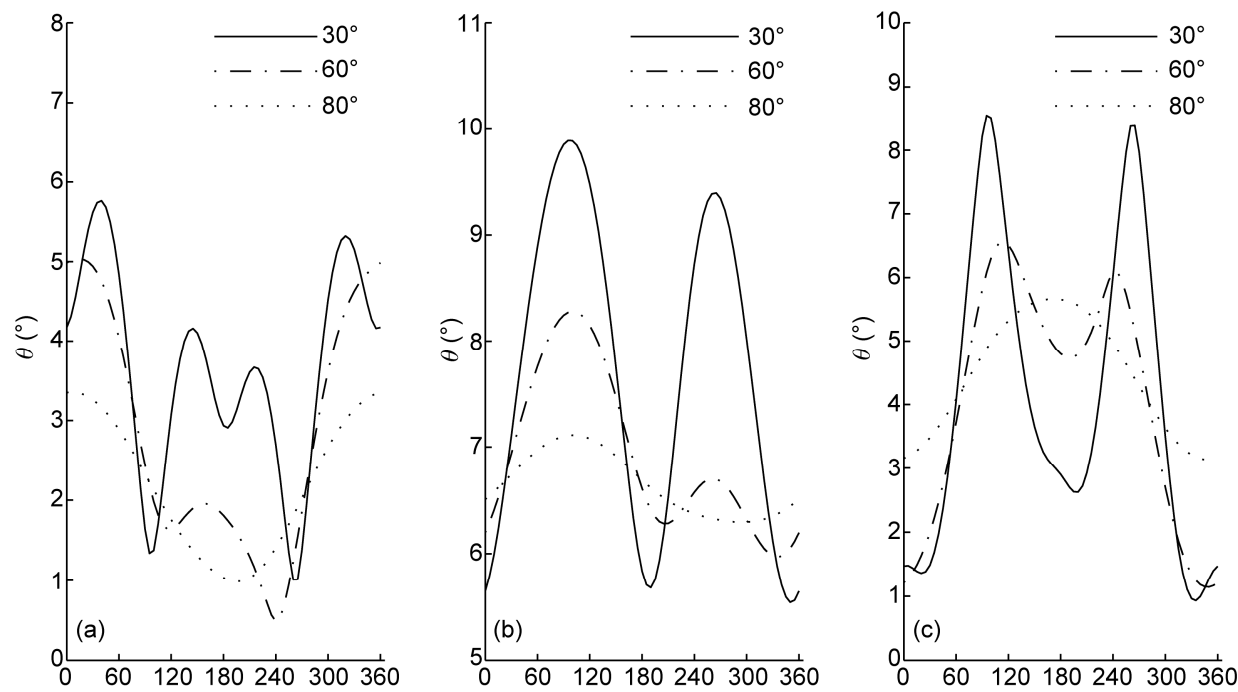

图 4 采用非正交矩阵的误差

比图 4 小很多, 可见, 即使误差上限很大, 当矢量 $\Delta S$ 与 $\boldsymbol{S}$ 的方向偏差很小时, 也可获得高测量精度.

针对矢量 $\boldsymbol{S}$ 各分量的实测误差值与其真实值的 不同比值, 表 1 给出了采用正交矩阵得到的误差上限 表, 表中 $k_{1}, k_{2}, k_{3}$ 表示矢量 $\boldsymbol{S}$ 各分量的实测误差值与 其真实值的比值.

\section{2 实测与结果分析}

本文通过分析实测太阳方向与其真实方向的吻 合度来验证定向方法的正确性和可行性. 太阳的真
实方向由太阳高度角和方位角求得, 太阳方位角取 正东为 $0^{\circ}$, 正南为 $90^{\circ}$, 太阳高度角和方位角由天文 公式(17)和(18) ${ }^{[16 \sim 19]}$ 计算得到:

$$
\begin{gathered}
\sin (h)=\sin (\psi) \sin (\delta)+\cos (\psi) \cos (\delta) \cos (t), \\
\sin (d)=\frac{\cos (\delta) \sin (t)}{\cos (h)},
\end{gathered}
$$

其中, $h$ 为太阳高度角, $d$ 为太阳方位角, $\psi$ 为当地纬 度, 太阳时角 $t$ 和赤纬角 $\delta$ 用文献[19]的公式计算. $(\cos (h) \cos (d), \cos (h) \sin (d), \sin (h))$ 为太阳真实方向. 

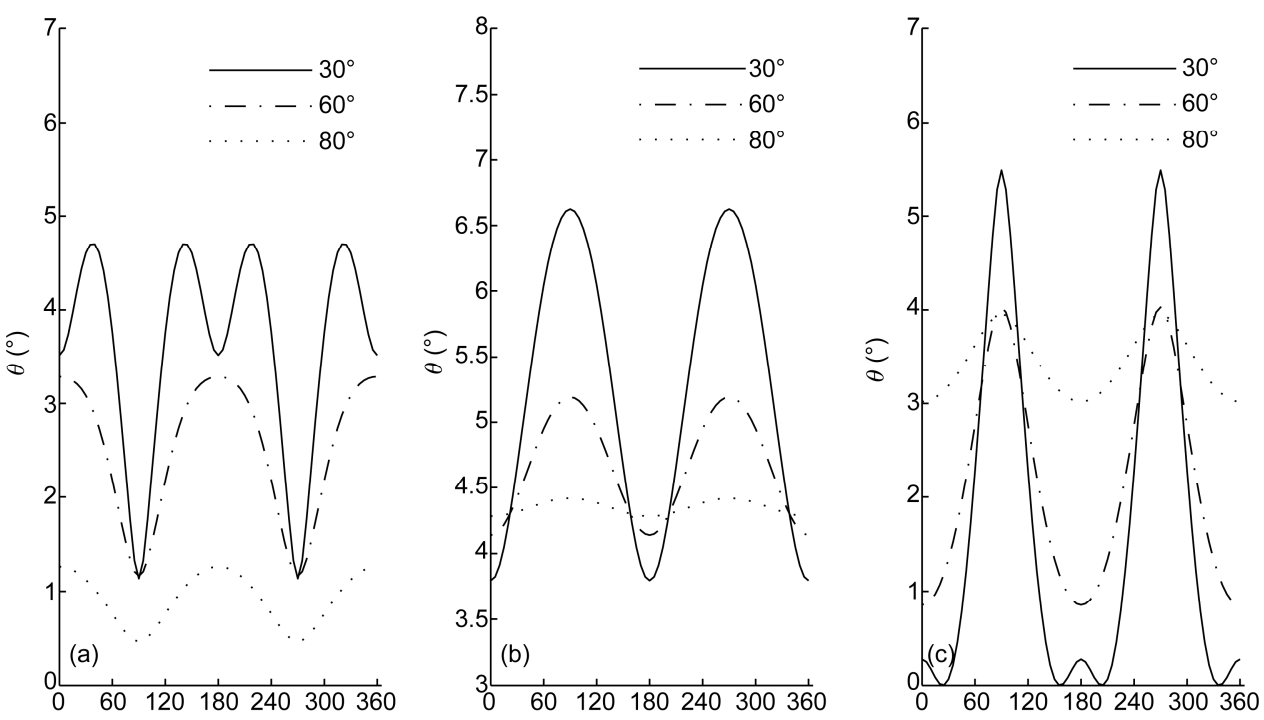

图 5 采用正交矩阵的误差
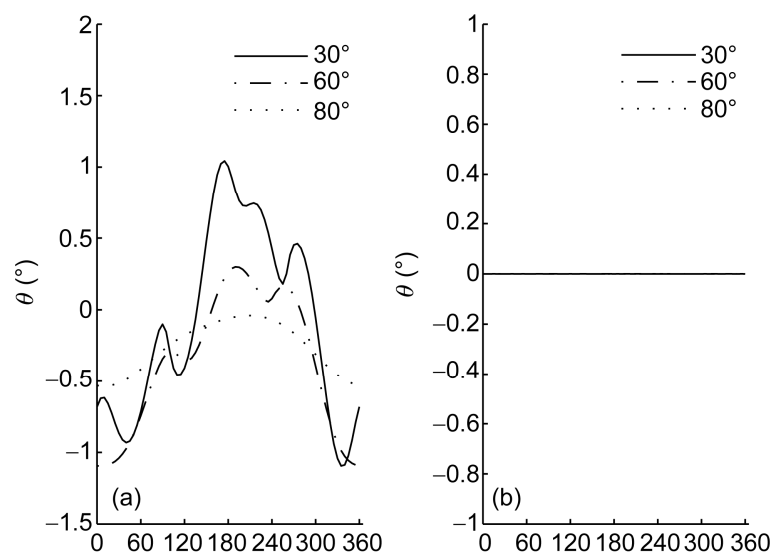

图 $6 S+\Delta S$ 和 $S$ 夹角与 $r+\Delta r$ 和 $r$ 夹角之差
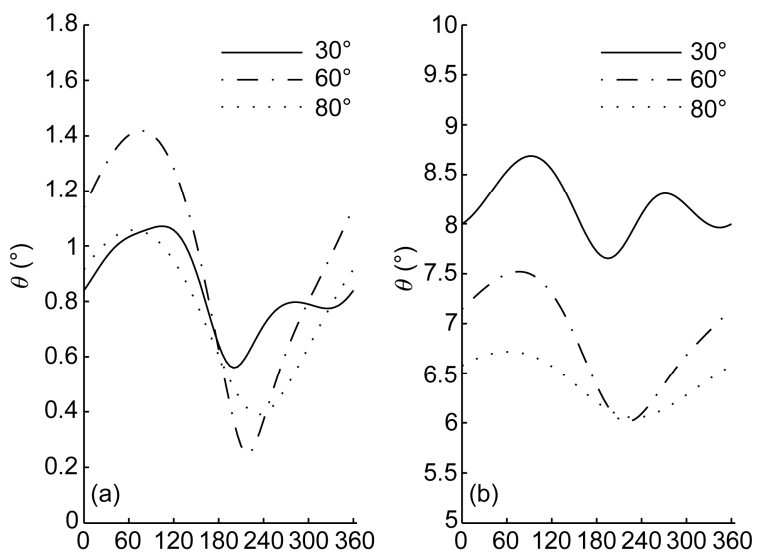

图 $7 \boldsymbol{k}$ 各分量间偏差较小的误差
表 1 正交矩阵的误差上限表

\begin{tabular}{cc}
\hline$k_{1}, k_{2}, k_{3}$ 的绝对值 & 误差上限 $(\theta)$ \\
\hline 0.2 & $11.537^{\circ}$ \\
0.1 & $5.7392^{\circ}$ \\
0.05 & $2.866^{\circ}$ \\
0.01 & $0.574^{\circ}$ \\
0.005 & $0.2865^{\circ}$ \\
\hline
\end{tabular}

实测装置为梯形台，梯形台底面和顶面为正方 形, 侧面与底面倾角为 $15.5^{\circ}$, 以梯形台中心为原点 建立空间直角坐标系, 如图 8 所示, 其 xoy 平面与地 平面平行且 $x$ 与 $y$ 正轴分别指向正东和正南, 太阳方 向为矢量 $\boldsymbol{E}$ 的方向. 光传感器为同型号太阳能电池, 分别安装在梯形台侧面和顶面，它们对同一太阳辐 照的实测输出偏差小于 $5 \%$. 因太阳能电池输出的短 路电流与光照强度近似线性变化 ${ }^{[20]}$, 实测中用其输 出的光电流代替太阳投射在梯形台各面上的能量.

测量时间为 2011 年 7 月 18 日, 测量装置安装在 楼顶, 周围视距较开阔, 测量地经纬度为 $103^{\circ} 59^{\prime}$, $30^{\circ} 35^{\prime}$, 天气为上午晴, 14 点半后多云. 实测结果如 图 9 所示, 图中 9(a)和(b)分别为太阳的真实方位角和 高度角, 图 9(c)为采用不同矩阵的太阳方向实测误差 曲线, 实线为采用梯形台上东、南、西三面的单位法 矢量构成的非正交矩阵测得的误差曲线, 虚线为采 用梯形台上顶、南、西三面的单位法矢量构成的非正 交矩阵测得的误差曲线, 其中, 顶、东、南、西四面 


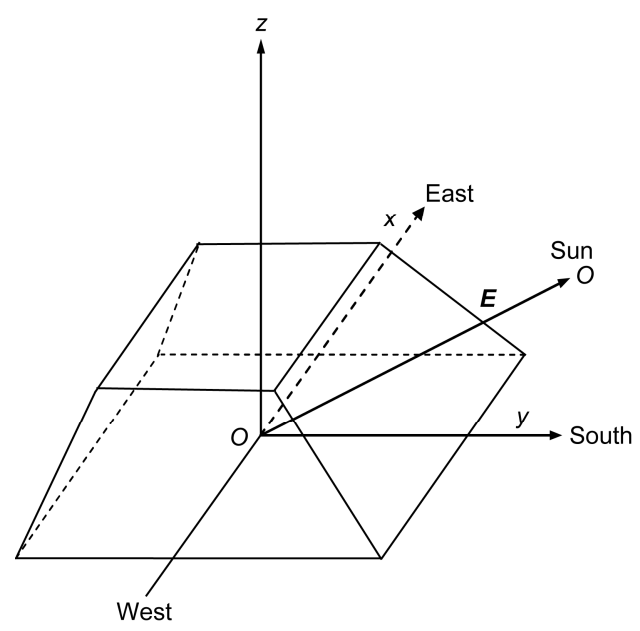

图 8 以梯形台建立的空间直角坐标系

的单位法向量分别为 $(0,0,1),(0.9636,0,0.2672),(0$, $0.9636,0.2672),(-0.9626,0,0.2672)$. 由光辐射源空 间定向原理可知两矩阵的测量范围为: 太阳高度 角 $>15.5^{\circ}$ 的任意方位角.

如图 9(c)所示, 太阳从方位角 $-10^{\circ} \sim 190^{\circ}$, 高度 角从 $25^{\circ} \sim 80^{\circ}$, 再从 $80^{\circ} \sim 25^{\circ}$ 运行期间, 太阳实测方向 与真实方向基本吻合. 从 $8: 40$ 到 $17: 20$, 除 $15: 30$ 左
右的多云时段，假设电池间的输出偏差为 $\pm 5 \%$; 实验 装置各面法线的几何偏差为 $\pm 1^{\circ}$, 不考虑太阳入射光 与各面法线夹角大于 $85^{\circ}$ 的情况, 由其产生的偏差为 $\pm 2 \%$ ；根据文献[21]，晴天条件下，仅在拉萨少部分 大气透明度高的地方, 当太阳高度角大于 $30^{\circ}$ 时太阳 直射与太阳总辐射才大于 $90 \%$, 由此可知, 各面大气 散射和地物反射产生的散射分量与太阳直射分量比 值大于 $10 \%$, 因此，设太阳散射分量与直射分量为 $10 \%$. 这样, 各面可能出现的偏差范围为 $17 \%$ 3\%, 对应正交矩阵其误差上限范围为 $\left[1.72^{\circ}, 9.79^{\circ}\right]$, 由于 实测矩阵为非正交矩阵, 因此, 实测误差上限将更大. 由仿真分析可知, 在不同高度角, 实际误差与误差上 限的差值随方位角波动, 在某些范围实际误差比误 差上限小很多. 分析图 9(c)可知, 在 8:40 到 17:20, 除 $15: 30$ 左右的时段, 太阳方向的实测误差小于 $4.4^{\circ}$, 其值在误差上限范围 $\left[1.72^{\circ}, 9.79^{\circ}\right]$ 内; 同时, 实测误 差随太阳高度角和方位角波动，在高度角为 $60^{\circ} \sim 80^{\circ}$ 的时段, 实测误差明显减小, 与仿真结果相符. 15 至 16 点时段, 因空中云层增加, 导致大气散射干扰增 大, 使得实测结果出现较大误差波动, 但因各面上大 气散射引入的偏差同向增大，使得误差增大幅度相
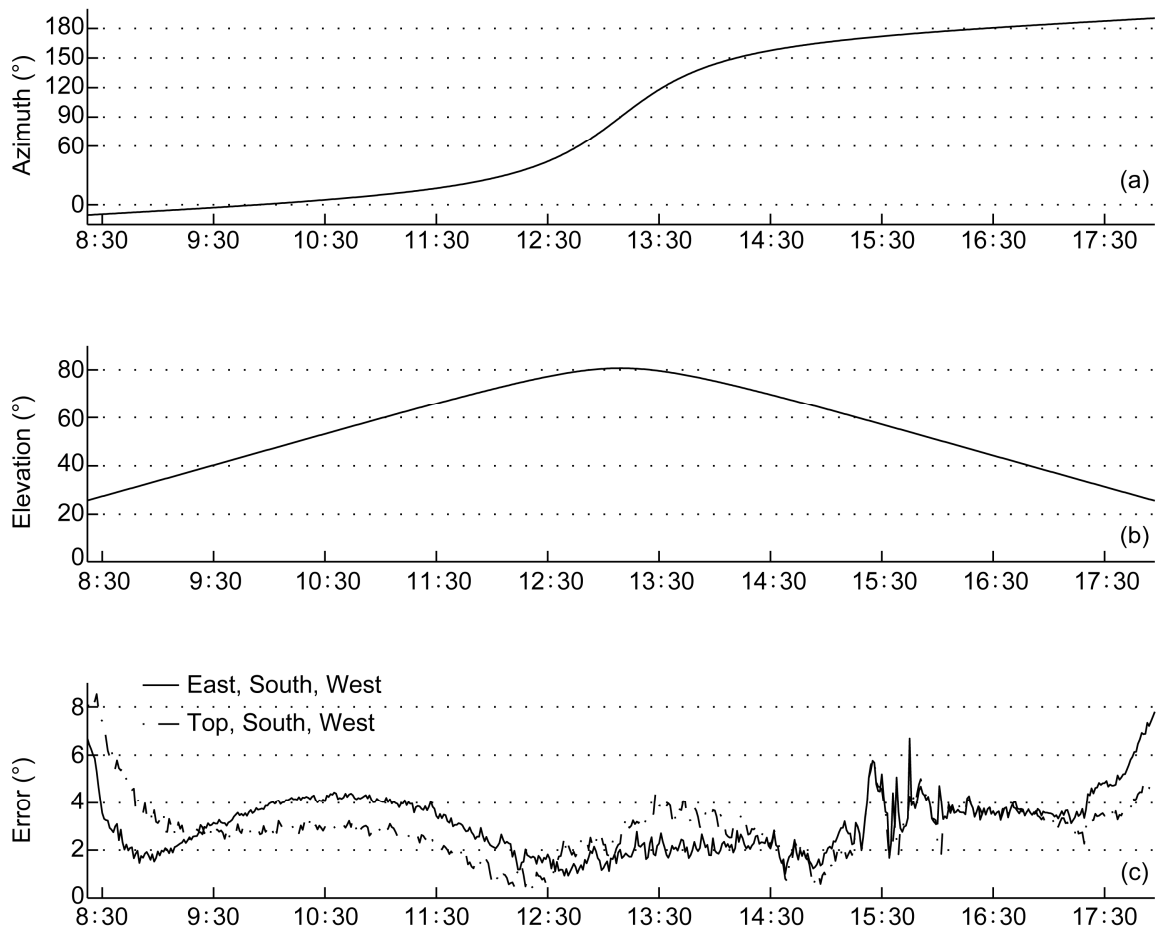

图 9 太阳方向的实测误差 
对较小, 其值小于 $6.7^{\circ} .8: 40$ 前与 17:20 后, 因太阳 直射能量较小, 电池输出值中的大气散射分量与太 阳直射分量的比值增大, 使得误差不断增大. 综上分 析, 实测结果与预期相符, 验证了本文方法的正确可 行性.

\section{6 结论}

基于推得的光辐射源的辐射能量和方向可用法 矢量不共面的 3 个平面的单位法矢量及光辐射源投 射在 3 个平面上的能量来求解的结论, 提出用多面体 对光辐射源空间定向的方法, 实现了 $360^{\circ}$ 全视场测 量, 解决了现有方法对 $180^{\circ}$ 以上视场定向时因测量 视场不全带来的探测系统体积、重量、功耗和成本增 加的问题. 实测与仿真结果表明该方法正确可行. 利 用矢量间的几何关系, 推导出了误差上限计算式, 解决了误差范围的确定问题. 仿真结果如下。

1) 当 3 个面的单位法矢量构成的测量矩阵非正
交时, 误差将被矩阵放大，而测量矩阵正交时，误差 与矩阵无关, 因此, 应用时应优先选择正交矩阵以提 高精度.

2) 当 3 个面上的能量构成的矢量与其误差构成 的矢量在方向上偏差足够小，即使误差上限很大，也 可获得高测量精度，该特性表明该方法在大噪声背 景下也可能实现高精度测量.

3) 对于正交测量矩阵，当多径等误差源在任一 测量面上产生的能量测量误差值与该面上能量真实 值的比值上限为 $5 \%$ 和 $1 \%$ 时, 测量精度分别优 $2.866^{\circ}$ 和 $0.574^{\circ}$.

为实现更高精度定向，实际应用时需解决传感 器的输出一致性问题及光辐射源多径干扰问题. 理 论上可增加多面体上被光辐射源同时直射的面来提 高抗多径干扰能力, 但实际应用还需进一步研究. 为 避免因安装造成的视场遮挡, 应用中可选如航天器 等被安装物体的外表作为测量多面体来实现 $360^{\circ}$ 全 视场测量.

\section{参考文献}

1 居鹤华，封红鹏，王贵财，等. 基于陀螺和太阳敏感器的月球车定向方法. 控制工程, 2011, 18: 313-317

2 王挺峰，郭劲. 线阵非成像式激光辐射源定向方法. 红外与激光工程, 2011, 40: 1649-1652

3 张世全，殷世民. 空中机动目标的红外单站被动定位算法分析. 激光与红外, 2011, 41: 1323-1326

4 Chang Y K, Kang S J, Lee B H. High-accuracy image centroiding algorithm for CMOS-based digital sun sensors. IEEE Sensors 2007 Conference, Atlanta GA, 28-31 Oct, 2007. 329-336

5 丁天怀, 毕研刚, 王鹏. 基于 CMOS APS 的微型数字式太阳敏感器. 清华大学学报(自然科学版), 2008, 48: 203-206

6 屠斌杰, 韩柯, 王吴, 等. 大视场数字式太阳敏感器设计. 传感技术学报, 2011, 24: 336-341

7 江洁, 温志明, 张广军, 等. 一种高性能太阳敏感器复合光学系统设计. 光学学报, 2008, 28: 344-348

8 席红霞. 大视场、高精度数字式太阳敏感器. 红外, 2003, 1: 25-29

9 郑志敏, 丁天怀. 小孔阵列式太阳敏感器的光学系统设计. 光学技术, 2006, 32: 240-243

10 陈非凡, 陈益峰. 采用光学非线性补偿的两轴微型太阳敏感器. 清华大学学报(自然科学版), 2004, 44: 197-200

11 Chen F F, Feng J, Hong Z W. Digital sun sensor based on the vernier measuring principle. Meas Sci Technol, 2006, (17): 2494-2498

12 尉志军, 刘晓军, 吕政欣. 一种新型太阳敏感器. 光电工程, 2010, 37: 123-126

13 詹亚锋, 马正新, 曹志刚. 现代微小卫星技术及发展趋势. 电子学报, 2000, 28: 102-106

14 林来兴. 分布式小卫星系统的技术发展与应用前景. 航天器工程, 2010, 19: 60-66

15 王炳忠, 莫月琴, 杨云. 现代气象辐射测量技术. 北京: 气象出版社, 2008. 19-30

16 Walraven R. Calculating the position of the sun. Sol Energy, 1978, 20: 393-397

17 Braun J E, Mitchell J C. Solar geometry for fixed and tracking surfaces. Sol Energy, 1983, 31: 439-444

18 Reda I, Andreas A. Solar position algorithm for solar radiation applications. Sol Energy, 2004, 76: 577-589

19 王炳忠, 刘庚山. 日射观测中常用天文参数的再计算. 太阳能学报, 1991, 12: 27-32

20 姜琳. 太阳能电池基本特性测定实验——个与能源利用有关的综合设计性实验. 大学物理, 2005, 24: 52-55

21 左大康，弓苒. 中国太阳直接辐射、散射和太阳总辐射间的关系. 地理学报, 1962, 28: 175-186 